\title{
Formación del pensamiento científico en alumnos de primer año de Odontología en dos universidades latino-americanas
}

\author{
Allana Pivovar*; Beatriz Castillo**; Cassius Carvalho Torres-Pereira*; Adriana Actis*** \\ * Programa de Posgrado en Odontología, Universidade \\ Federal do Paraná \\ * Asesoría Pedagógica. Facultad de Odontología. \\ Universidad Nacional de Córdoba \\ *** Cátedra B de Anatomía, Facultad de Odontología, \\ Universidad Nacional de Córdoba
}

Recibido el 27/03/2019. Aceptado el 02/06/2019.

\begin{abstract}
RESUMEN
El objetivo del estudio fue analizar el desarrollo del pensamiento científico, en alumnos de grado del primer año, a partir de la inclusión de la asignatura metodología científica en el inicio de la carrera de Odontología en dos universidades latinoamericanas (Brasil y Argentina). La muestra estuvo constituida por 144 estudiantes. Si bien se ha detectado el inicio de la formación del pensamiento científico en los alumnos que integraron la muestra, se requiere un desarrollo más completo, gradual y complejo de esta competencia para el logro de las líneas directrices establecidas por las dos universidades para un profesional odontólogo.

Descriptores: Metodología Científica. Educación. Asociación de Universidades del Grupo Montevideo.
\end{abstract}

\section{INTRODUCCIÓN}

La ciencia se origina en la capacidad del individuo de indagar sobre la realidad alrededor, de formular cuestionamientos y de establecer reflexiones críticas frente a las más diversas situaciones, buscando cambiarlas ${ }^{1}$. La formación en ciencia es fundamental para promover el desarrollo científico y tecnológico de un país. La ciencia en conjunto con la educación puede ser comprendida como las herramientas más básicas en el proceso de democratización, pues contribuyen a mejorar las condiciones de vida para la población ${ }^{1,2}$. La Conferencia Mundial de Educación Superior promovida por la UNESCO 
en París (1998) tuvo una importante conclusión: no hay condiciones de una nación moderna con desarrollo social y económico si no tiene una base científica y tecnológica ${ }^{3}$. Siendo así, el desarrollo del pensamiento científico es un rasgo fundamental en la educación superior ${ }^{2,4,5}$.

El pensamiento científico puede definirse como una actitud o disposición del investigador en la búsqueda de soluciones serias, y con los métodos adecuados, para el problema que se enfrenta. Esta actitud no es innata en las personas sino que se conquista durante la vida y puede ser aprendida. Se traduce como una mente crítica, objetiva y racional. La mente crítica evita la aceptación de lo que es fácil y superficial. La conciencia objetiva implica la ruptura con las posiciones subjetivas. Por lo tanto, la racionalidad mantiene los objetos de la investigación en el campo de la realidad física, no espiritual ${ }^{6}$.

El conocimiento científico en el contexto de las ciencias fácticas se caracteriza por ser racional, objetivo, analítico, claro y preciso, notificable y verificable, dependiente de la investigación metódica, sistemática, acumulativa, falible, general, explicativa, predictiva, abierta y útil ${ }^{7}$. Los principales rasgos que comprenden al pensamiento científico son la confiabilidad, el dinamismo y la verificación. Un conocimiento científico es válido y confiable cuando el método científico opera como una instancia reguladora y evaluadora de la producción científica, y por lo tanto, no está exento de error y puede ser refutado por nuevas investigaciones ${ }^{8}$. Constituye un conocimiento falible, no definitivo, absoluto o final y, por lo tanto, es cuasi preciso ya que nuevas propuestas y técnicas de desarrollo pueden reformular el acervo de la teoría existente ${ }^{7}$. Finalmente, es un saber verificable. Son construidos a partir de referentes empíricos que sustentan la validez de su verdad ${ }^{8}$.
La incorporación del método científico en el curriculum, ineludible en la educación superior, se fundamenta en la necesidad y el compromiso de generar, divulgar y utilizar conocimientos, prácticas y tecnologías que conformen un recurso humano capaz de integrarse en las diferentes modalidades de la práctica en salud y transformarlas con una actitud crítica, reflexiva e inquisitiva ${ }^{2,4,5}$. La inclusión de actividades de investigación dependerá de la concepción que tengan las instituciones educativas sobre su papel en la sociedad, su compromiso y su conocimiento de la realidad $\operatorname{social}^{2,5}$. A pesar de que la enseñanza de la metodología científica es una decisión institucional, es importante considerar la percepción del alumno, actor principal del proceso, respecto de este aprendizaje ${ }^{9}$. En este aspecto, no se han encontrado muchos estudios de tipo cualitativo en cuanto a la incorporación de la metodología científica en la carrera de Odontología?.

Este estudio es fruto de una experiencia de movilidad de estudiantes de posgrado que pretende lograr un intercambio cultural y académico. El Programa Escala de Estudiantes de Posgrado de la Asociación de Universidades del Grupo Montevideo (AUGM) promueve la cooperación y la integración de las Universidades que la conforman, así como la internacionalización de la educación superior, a través de la promoción de la movilidad de los estudiantes regulares de maestrías y doctorados, para cursar un período académico en otra universidad miembro de esta asociación de un país distinto al suyo ${ }^{10}$.

El objetivo del presente estudio fue analizar el desarrollo del pensamiento científico en alumnos de grado a partir de la inclusión de la asignatura Metodología Científica en el inicio de la carrera de Odontología en dos universidades latinoamericanas: Universidad Federal de Paraná 
(UFPR) (Brasil) y Universidad Nacional de Córdoba (UNC) (Argentina). Al establecer una comparación entre las dos universidades, este estudio pretendió evidenciar las dificultades y oportunidades que surgen cuando se incluye el conocimiento sobre el pensamiento científico.

\section{METODOLOGÍA}

Este estudio, que fue aprobado por el Comité de Ética e investigación de la UFPR (CAAE: 51699815.8.0000.0102), es de tipo transversal, descriptivo y analítico. En primer lugar, se hizo un análisis descriptivo/ comparativo de los programas académicos de las asignaturas Metodología Científica (UFPR) y Metodología de la Investigación en Ciencias de la Salud (UNC), los que se ajustan a la normativa nacional de las carreras de Odontología de cada país.

Luego, se desarrolló un cuestionario destinado a recoger información sobre opiniones de los alumnos acerca del conocimiento científico, su aprendizaje y aplicación a la formación de estudiantes de odontología. Este cuestionario fue basado en otro, previamente utilizado y calibrado, desarrollado por Shirahatti (2010) en India ${ }^{11}$. El cuestionario autoadministrado fue validado en una muestra correspondiente al $10 \%$ del total de inscritos en las disciplinas en estudio de cada universidad. En la UNC, la versión en español fue validada en un grupo de 59 alumnos regulares de la asignatura Metodología de la Investigación en Ciencias de la Salud. En tres enunciados, cuatro alumnos tuvieron dificultades de comprensión, totalizando apenas un $6,7 \%$ de la muestra $(n=4 / 59)$. La versión en portugués fue aplicada en 10 alumnos que habían aprobado la disciplina Metodología Científica en la UFPR en el primer semestre del año 2015. Esta versión presentó dos enunciados que no pudieron ser comprendidos por el $10 \%$ de la muestra $(n=1 / 10)$. Sobre la base de esta validación, se modificaron los aspectos que dificultaban la comprensión por parte de los alumnos.

La población del estudio estuvo constituida por alumnos de primer año, regulares en las dos asignaturas mencionadas en la UNC y UFPR que la cursaban por primera vez y que estaban presentes el día de la administración del instrumento (último día del cronograma de la disciplina en el que los alumnos de la UNC realizaron la presentación de sus trabajos finales y los de la UFPR efectuaron un examen teórico). Los estudiantes fueron invitados a participar de manera voluntaria y anónima y quienes aceptaron debieron completar el formulario de consentimiento informado (UFPR).

Para la conformación de la muestra en la UNC, se seleccionaron por sorteo aleatorio 104 de 204 participantes (presentes al momento de administrar el instrumento) ( $\mathrm{n}=104 ; 51 \%)$. Por otra parte, en la UFPR se incluyó la totalidad de los alumnos presentes $(n=40 ; 100 \%)$ debido a la gran discrepancia en el número de estudiante inscritos en la carrera de las dos universidades (UNC=700; UFPR=45). Para cada enunciado de la escala Likert se aplicó la siguiente fórmula para la obtención del score final de cada enunciado: $\mathrm{f} \times 6+\mathrm{f} \times 5+\mathrm{f} \times 4+\mathrm{f} \times 3+\mathrm{f} \times 2+\mathrm{f} \times 1 / \mathrm{n} ; \mathrm{f}=$ frecuencia de respuesta e $n=$ número total de participantes.

\section{Metodología Científica en UFPR (Brasil)}

En marzo de 2002 se constituyeron en Brasil las Directrices Curriculares para las carreras en general y para la Odontología en particular, orientadas a cambios que involucran posturas teórico-metodológicas de enseñanza-aprendizaje con reflejo sobre la organización curricular. Los proyectos pedagógicos deberían estar orientados a la formación de un profesional más comprometido con la ciencia y los problemas sociales ${ }^{12}$. Entre las características destacadas en las directrices 
curriculares de odontología, los alumnos deben poseer las competencias y habilidades para evaluar, sistematizar y decidir por las conductas más adecuadas, basadas en la evidencia científica, además de conocer métodos y técnicas de investigación y elaboración de trabajos científicos, analizar e interpretar los resultados relevantes de trabajos de investigación experimentales, epidemiológicos y clínicos, participar de investigación científica sobre enfermedades de la cavidad bucal y estar preparado para aplicar los resultados de investigación para la promoción de la salud. Esta diretriz también incluye un trabajo de finalización de la carrera que el alumno deberá desarrollar con orientación de un docente y la inclusión de una asignatura sobre metodología científica como parte de la formación básica ${ }^{13}$.

La asignatura Metodología Científica fue institucionalizada en Odontología de la UFPR en el año 2008 y está ubicada en el primer semestre de la carrera. La propuesta metodológica consiste en una actividad práctica, de estudio dirigido en la que los alumnos plantean un problema de investigación y efectúan una búsqueda bibliográfica para luego hacer un análisis crítico y presentar los resultados en seminarios evaluativos de corta duración.

\section{Metodología de Investigación en Ciencias de la Salud en UNC (Argentina)}

La Resolución 1413/08 del Ministerio de Educación de la Nación Argentina determina los contenidos curriculares básicos para las carreras de Odontología de todo el país, los que deben ser incorporados obligatoriamente por ser considerados esenciales para el reconocimiento nacional del título de odontólogo. Dentro de esos contenidos se incluyen epistemología e introducción a la investigación científica.

Las líneas directrices del plan de estudios de la carrera de Odontología contemplan que sus egresados sean personas competentes en el análisis e interpretación de los resultados relevantes de las investigaciones clínicas, epidemiológicas y experimentales, que ejerzan una práctica odontológica basada en la evidencia científica y que tiendan a la mejora de la calidad de vida de las personas y las comunidades. El plan de estudios no contempla la realización de un trabajo final.

La disciplina Metodología de la Investigación en Ciencias de la Salud fue institucionalizada en la Facultad de Odontología de la UNC en el año 2010 y está incluida en el ciclo básico. La asignatura propone a los alumnos, como estrategia de enseñanza, una experiencia de construcción y desarrollo de un proyecto de investigación, donde los estudiantes -en forma grupal- plantean desde el problema de investigación hasta el análisis de los datos obtenidos mediante herramientas sencillas, generalmente encuestas, y aplicadas a poblaciones de bajo riesgo, previo consentimiento informado. Todo el desarrollo del proyecto es apoyado por clases teóricas y es guiado por docentes durante todo el cuatrimestre. Finalmente, los grupos de alumnos entregan un trabajo escrito y presentan, de manera oral y mediante un póster, los resultados finales del trabajo. La tabla 1 presenta las características organizativas de las asignaturas Metodología científica en UFPR y Metodología de la investigación en Ciencias de la Salud (UNC) y la tabla 2 muestra una comparación de sus contenidos.

\section{RESULTADOS}

Participaron de la investigación 104 alumnos de la UNC - 26 hombres (25\%) y 78 mujeres (75\%) - y 40 alumnos de la UFPR - 10 hombres (25\%) y 30 mujeres (75\%). La media de edad de los participantes fue de 19 años (variando de 17 a 34 años) en los dos países. La tabla 3 y los cuadros I a IV presentan los resultados acerca de las opiniones y conocimientos de los alumnos sobre el pensamiento científico. Los resultados de la mencionada tabla fueron analizados sobre la base 
de tres categorías de análisis previamente diseñadas:

a. Relación entre pensamiento científico y ciencia: se observaron similitudes en las respuestas de los alumnos de las dos universidades. Para los estudiantes, pensar científicamente implica el desarrollo de estrategias que permitan comprender el mundo a través de la ciencia. Ellos también reconocen las implicancias sociales de la ciencia y el respeto por la vida y el ambiente.

b. Aprendizajes logrados en la asignatura en estudio: según los participantes, las asignaturas sobre metodología de investigación permitieron adquirir un trabajo metódico y rigor intelectual como aporte a su proceso de estudio. Además, ellos reconocen la posibilidad de identificar un problema y leer un artículo científico aunque no escribirlo.

c. Relación entre el pensamiento científico y la práctica profesional: en general, los alumnos consideran que, para quienes se dediquen en el futuro a la actividad profesional clínica, no es necesario profundizar, de manera gradual, los contenidos relacionados a la investigación; esto sería apropiado para quienes elijan ser investigadores. Por otra parte, los participantes sostienen que el razonamiento clínico que el profesional aplica en su tarea cotidiana no requiere las mismas etapas que el pensamiento científico utilizado en un trabajo de investigación.

Tabla 1. Características organizativas de las asignaturas estudiadas en las dos universidades

\begin{tabular}{|c|c|c|}
\hline Característica & UFPR (Brasil) & UNC (Argentina) \\
\hline Duración & 4 meses & 4 meses \\
\hline $\begin{array}{l}\text { Ubicación de la } \\
\text { asignatura en el } \\
\text { Plan de Estudio }\end{array}$ & $\begin{array}{l}\text { Primer año de la carrera en el } \\
\text { primer cuatrimestre }\end{array}$ & $\begin{array}{l}\text { Primer año de la carrera en el segundo } \\
\text { cuatrimestre }\end{array}$ \\
\hline $\begin{array}{l}\text { Total de horas de } \\
\text { la asignatura }\end{array}$ & 30 horas & 64 horas \\
\hline $\begin{array}{l}\text { Actividades } \\
\text { teóricas }\end{array}$ & $\begin{array}{l}20 \text { horas ( } 2 \text { horas semanales); } \\
\text { obligatorias; modalidad } \\
\text { expositiva }\end{array}$ & $\begin{array}{l}16 \text { horas ( } 1 \text { hora semanal); no obligatorias; } \\
\text { modalidad expositiva }\end{array}$ \\
\hline $\begin{array}{l}\text { Actividades } \\
\text { prácticas }\end{array}$ & $\begin{array}{l}10 \text { horas } \\
\text { (1 hora semanal); } \\
\text { obligatorias; } \\
\text { Estudio dirigido y } \\
\text { presentación de seminarios } \\
\text { evaluativos de } 15 \text { minutos, } \\
\text { desarrollado en grupos de } 6 \\
\text { alumnos; } \\
\text { Utilización de Plataforma } \\
\text { Moodle }\end{array}$ & $\begin{array}{l}48 \text { horas } \\
\text { ( } 3 \text { horas semanales); obligatorias; } \\
\text { Modalidad taller: grupos de } 3-4 \text { alumnos que } \\
\text { diseñan y desarrollan un proyecto de } \\
\text { investigación; } \\
\text { Utilización de Plataforma Moodle }\end{array}$ \\
\hline $\begin{array}{l}\text { Número de } \\
\text { profesores }\end{array}$ & 1 profesor titular. & 2 profesores titulares, 12 profesores asistentes. \\
\hline $\begin{array}{l}\text { Número total de } \\
\text { alumnos }\end{array}$ & 45 & 700 \\
\hline Evaluación & $\begin{array}{l}\text { Dos evaluaciones teóricas y } \\
\text { un trabajo práctico } \\
\text { (seminario) }\end{array}$ & $\begin{array}{l}\text { Evaluación del proyecto de investigación en } \\
\text { dos momentos: fase de proyecto y fase final } \\
\text { (resultados/conclusiones finales) }\end{array}$ \\
\hline
\end{tabular}

UNC: Universidad Nacional de Córdoba. UFPR: Universidad Federal de Paraná. 
Tabla 2. Comparación de los contenidos de las asignaturas estudiadas en las dos universidades

\begin{tabular}{|c|c|c|}
\hline Contenidos & UFPR & UNC \\
\hline $\begin{array}{l}\text { Importancia del conocimiento científico dentro de la carrera de Odontología y Ciencias de la } \\
\text { Salud }\end{array}$ & $\mathrm{Si}$ & $\mathrm{Si}$ \\
\hline Características del conocimiento científico & $\mathrm{Si}$ & No \\
\hline Búsqueda y selección de información en bancos de datos & $\mathrm{Si}$ & $\mathrm{Si}$ \\
\hline Selección de estrategias metodológicas cuantitativas o cualitativas según el objeto a investigar & $\mathrm{Si}$ & $\mathrm{Si}$ \\
\hline $\begin{array}{l}\text { Establecimiento de la dinámica en el proceso de recolección, selección del diseño y análisis de la } \\
\text { información }\end{array}$ & $\mathrm{Si}$ & $\mathrm{Si}$ \\
\hline Análisis crítico e interpretación de resultados & $\mathrm{Si}$ & $\mathrm{Si}$ \\
\hline Identificación de las partes que componen un trabajo científico & $\mathrm{Si}$ & $\mathrm{Si}$ \\
\hline Odontología basada en la evidencia & $\mathrm{Si}$ & $\mathrm{Si}$ \\
\hline Elaboración de un informe de comunicación científica & No & $\mathrm{Si}$ \\
\hline $\begin{array}{l}\text { Reconocimiento de distorsiones informativas y conceptuales sugeridas en las propagandas de } \\
\text { productos y tecnologías }\end{array}$ & $\mathrm{Si}$ & No \\
\hline Orientación acerca del trabajo final de la carrera & $\mathrm{Si}$ & No \\
\hline
\end{tabular}

UNC: Universidad Nacional de Córdoba. UFPR: Universidad Federal de Paraná.

Tabla 3. Opiniones de los alumnos acerca del conocimiento científico

\section{Como alumno y después de haber cursado la asignatura considero que: \\ Score UNC UFPR}

a. Los conocimientos aprendidos en metodología de investigación me permitieron adquirir e incorporar, a mi proceso de estudio en la universidad, rigor intelectual, trabajo metódico, interés por averiguar más sobre los temas de estudio.

b. Lo que aprendí en primer año, sobre metodología de investigación en ciencias de la salud, es suficiente para la formación de un odontólogo.

c. Estoy en condiciones de identificar un problema que pueda ser objeto de investigación.

d. Me siento capaz de leer un artículo científico de manera crítica y evaluar el nivel de evidencia de los resultados y conclusiones.

e. Me siento capaz de escribir un texto científico (informe de laboratorio, elaboración de protocolo, etc.) teniendo en cuenta la terminología, las reglas del lenguaje de las ciencias.

f. Para el estudiante que, en el futuro, se va a dedicar a la actividad profesional clínica (consultorio, hospital, etc.), es necesario ir profundizando, en forma gradual a través de los años de formación de la carrera, los contenidos relacionados a la investigación.

g. Para el estudiante que, en el futuro, se va a dedicar a la investigación clínica, es necesario ir profundizando de manera gradual a través de los años de formación de la carrera, contenidos relacionados a la investigación.

h. Cuando egrese de la carrera usaré los procesos de investigación científica en la práctica profesional clínica (consultorio, hospital, etc.).

i. Mi manera de entender el conocimiento científico ha cambiado totalmente con respecto a lo que pensaba antes.

j. Pensar científicamente es desarrollar estrategias para relacionarme con el mundo a través de las ciencias.

k. Pensar científicamente es tener conocimientos científicos.

1. El razonamiento clínico que ejerce el profesional odontólogo, en su tarea cotidiana con los pacientes, requiere de las mismas etapas que el pensamiento científico utilizado en una investigación.

m. Como ciudadano y estudiante debo ser consciente de las implicancias sociales de la ciencia y decidir por aquellos conocimientos que contribuyan a respetar la vida y el ambiente. UNC: Universidad Nacional de Córdoba. UFPR: Universidad Federal de Paraná.

\section{$5,12 \quad 5,6$}

$4,02 \quad 3,6$

$5,2 \quad 5,1$

$5 \quad 5,07$

$4,27 \quad 4,5$

$5,29 \quad 5,3$

$5,75 \quad 5,65$

$4,74 \quad 4,87$

$4,73 \quad 5,62$

$5,25 \quad 5,37$

$4,54 \quad 4,22$

$4,68 \quad 4,37$

$5,62 \quad 5,85$ 
Ante la pregunta de cómo contribuye la ciencia en el desarrollo económico y tecnológico de un país (cuadro 1), en ambas universidades y de manera similar, se observa que los mayores porcentajes de respuestas destacan la contribución de la investigación a la mejora en las condiciones de vida y a la obtención de nuevos conocimientos. Sin embargo, se encontró un mayor porcentaje en la universidad brasileña en cuanto a la relación entre la ciencia y el desarrollo tecnológico (UFPR 31\%; UNC 13\%).

Las categorías de análisis que surgen de la información contenida en los cuadros 2 y 3 ponen de manifiesto los rasgos del conocimiento científico - confiablidad, dinamismo y verificación - con diferencias en las dos universidades. El $50 \%$ y el $26 \%$ de los alumnos de la UFPR y de la UNC, respectivamente, consideran que el conocimiento científico puede ser refutado, así como también el 33\% (UFPR) y el $14 \%$ (UNC) de los estudiantes relacionan confiabilidad del conocimiento con el método científico y el $12 \%$ (UNC) y el $2 \%$ (UFPR) vinculan la confiabilidad con el argumento de autoridad. Además, el 33\% (UNC) y el $21 \%$ (UFPR) de los alumnos respondieron que el conocimiento nunca está acabado. A pesar de que un alto porcentaje de alumnos de las dos universidades reconoce la confiabilidad del conocimiento científico y su contribución al desarrollo de un país, no puede dar fundamentos al respecto.

Cerca del $70 \%$ de los alumnos en ambas universidades concuerdan con la ubicación actual de la asignatura, es decir en el segundo cuatrimestre de la carrera (cuadro 4). El resto (UFPR 22,5\% y UNC 10,4\%) considera que sería más provechosa esta asignatura a partir del tercer cuatrimestre o más próxima a la preparación del trabajo final de la carrera en el caso de la UFPR. Además, los estudiantes de esta última destacan, en un $25 \%$, la necesidad de retomar y profundizar la temática en otros espacios curriculares, en tanto que sólo el $2 \%$ de los alumnos de la UNC comparten esta opinión.

Por otra parte, los alumnos destacan distintos aspectos relacionados con la asignatura: mejora en las estrategias de aprendizaje (39\% UNC y $16 \%$ UFPR) y en la capacidad de lectura de artículos científicos (32\% UFPR y $2 \%$ UNC).

Finalmente, el $100 \%$ y $39 \%$ de los alumnos de UFPR y UNC, respectivamente, conocen las oportunidades de participación en investigación que ofrece cada universidad. Sin embargo, los alumnos no manifestaron gran interés en realizar actividades de investigación (54\% UNC y $35 \%$ UFPR) ya que prefieren dedicarse a otras tareas, preferentemente vinculadas a la práctica profesional clínica.

\section{DISCUSIÓN}

En este artículo se propuso analizar el desarrollo del pensamiento científico, en alumnos de grado, a partir de la inclusión de la asignatura metodología científica en el inicio de la carrera de Odontología en dos universidades latinoamericanas: Universidad Federal de Paraná (UFPR) (Brasil) y Universidad Nacional de Córdoba (UNC) (Argentina). Al establecer una comparación entre las dos universidades, este estudio pretendió evidenciar las dificultades y oportunidades que surgen cuando se incluye el conocimiento sobre el pensamiento científico.

Los planes de estudio de la carrera de Odontología de las dos universidades contemplan la inclusión de metodología científica y el desarrollo de competencias para un ejercicio profesional basado en la evidencia científica.

En general, los alumnos participantes - de primer año de la carrera de Odontología reconocen que el pensamiento científico implica el desarrollo de estrategias que permitan comprender el mundo, que la ciencia contribuye 
Cuadro 1. ¿Cómo contribuye la ciencia en el desarrollo económico y tecnológico del país?

\begin{tabular}{|c|c|c|c|c|c|}
\hline \multirow{2}{*}{ Categoría } & \multirow{2}{*}{ Explicación } & \multicolumn{2}{|c|}{$\begin{array}{c}\text { UNC } \\
\text { (Argentina) }\end{array}$} & \multicolumn{2}{|c|}{$\begin{array}{l}\text { UFPR } \\
\text { (Brasil) }\end{array}$} \\
\hline & & f & $\%$ & f & $\%$ \\
\hline \multirow{4}{*}{$\begin{array}{l}\text { Mejora las } \\
\text { condiciones de vida } \\
\text { (educación y salud) }\end{array}$} & $\begin{array}{l}\text { "Propone interrogantes y soluciones a problemas } \\
\text { de la población que permiten mejorar las } \\
\text { condiciones de vida" }\end{array}$ & 15 & $13 \%$ & 5 & $13 \%$ \\
\hline & $\begin{array}{l}\text { "Contribuye a mejorar la educación y brinda } \\
\text { información a los ciudadanos; es esencial para la } \\
\text { formación integral de la persona" }\end{array}$ & 4 & $4 \%$ & 3 & $8 \%$ \\
\hline & $\begin{array}{l}\text { "Promueve la salud al posibilitar nuevos } \\
\text { tratamientos de las enfermedades y desarrollos } \\
\text { tecnológicos (equipamientos, p. ej), incentiva el } \\
\text { desarrollo de políticas y la asignación de recursos } \\
\text { económicos para su preservación" }\end{array}$ & 10 & $9 \%$ & 3 & $8 \%$ \\
\hline & $\begin{array}{l}\text { "Posibilita una mejor comprensión del país y del } \\
\text { mundo, con lo que se pueden introducir cambios } \\
\text { positivos" }\end{array}$ & 4 & $4 \%$ & 3 & $8 \%$ \\
\hline \multirow{4}{*}{$\begin{array}{l}\text { Promueve el } \\
\text { desarrollo } \\
\text { científico y } \\
\text { tecnológico }\end{array}$} & $\begin{array}{l}\text { "Permite generar nuevas tecnologías que } \\
\text { conducen a una independencia económica y } \\
\text { tecnológica del país" }\end{array}$ & 4 & $4 \%$ & 2 & $5 \%$ \\
\hline & $\begin{array}{l}\text { "Contribuye a mejorar la industria y la } \\
\text { agronomía" }\end{array}$ & 2 & $2 \%$ & 1 & $3 \%$ \\
\hline & $\begin{array}{l}\text { "El desarrollo de nuevas tecnologías y } \\
\text { conocimientos permite la prestación de servicios a } \\
\text { otros países (exportaciones) e incentiva las } \\
\text { relaciones internacionales, al tiempo que atraen } \\
\text { capitales extranjeros" }\end{array}$ & 3 & $3 \%$ & 2 & $5 \%$ \\
\hline & "Produce eficiencia y productos de mejor calidad" & 5 & $4 \%$ & 7 & $18 \%$ \\
\hline $\begin{array}{l}\text { Brinda } \\
\text { oportunidades } \\
\text { laborales }\end{array}$ & $\begin{array}{l}\text { "El estado brinda empleo a los investigadores, con } \\
\text { lo que la ciencia genera propuestas de trabajo" }\end{array}$ & 4 & $4 \%$ & 0 & 0 \\
\hline $\begin{array}{l}\text { Se generan nuevos } \\
\text { conocimientos }\end{array}$ & $\begin{array}{l}\text { "Brinda información para el progreso y el avance } \\
\text { del país" }\end{array}$ & 21 & $19 \%$ & 6 & $15 \%$ \\
\hline Imprecisas & $\begin{array}{l}\text { "Contribuye positivamente, pero no se } \\
\text { fundamenta la contribución" }\end{array}$ & 33 & $29 \%$ & 8 & $20 \%$ \\
\hline $\begin{array}{l}\text { No sabe la } \\
\text { respuesta }\end{array}$ & ---- & 1 & $1 \%$ & 0 & 0 \\
\hline \multirow{3}{*}{ Otras } & $\begin{array}{l}\text { "La ciencia en Argentina no está desarrollada y } \\
\text { recibe poco incentivo gubernamental" }\end{array}$ & 3 & $3 \%$ & 0 & 0 \\
\hline & "Otorga mayor riqueza y poder a los países" & 1 & $1 \%$ & 0 & 0 \\
\hline & $\begin{array}{l}\text { "Contribuye a la sociedad y al ambiente, siempre } \\
\text { que sea aplicada adecuadamente" }\end{array}$ & 2 & $2 \%$ & 0 & 0 \\
\hline
\end{tabular}

UNC: Universidad Nacional de Córdoba. UFPR: Universidad Federal de Paraná. 
Cuadro 2. ¿En qué medida es confiable un conocimiento científico?

\begin{tabular}{|c|c|c|c|c|c|}
\hline \multirow[t]{2}{*}{ Categoría } & \multirow[t]{2}{*}{ Explicación } & \multicolumn{2}{|c|}{$\begin{array}{c}\text { UNC } \\
\text { (Argentina) }\end{array}$} & \multicolumn{2}{|c|}{$\begin{array}{c}\text { UFPR } \\
\text { (Brasil) }\end{array}$} \\
\hline & & $\mathbf{f}$ & $\%$ & $\mathbf{f}$ & $\%$ \\
\hline \multirow[t]{2}{*}{$\begin{array}{l}\text { Aplicando el } \\
\text { método científico }\end{array}$} & $\begin{array}{l}\text { "Cuando el investigador no está patrocinado y no } \\
\text { modifica los resultados para obtener aquellos } \\
\text { deseados por el patrocinador. Siempre se debe } \\
\text { mantener la ética" } \\
\text { "Cuando las investigaciones y los análisis son } \\
\text { hechos con responsabilidad y seriedad" }\end{array}$ & 3 & $3 \%$ & 0 & \\
\hline & $\begin{array}{l}\text { "Cuando el método científico opera como una } \\
\text { instancia reguladora y evaluadora de la } \\
\text { producción científica" }\end{array}$ & 16 & $14 \%$ & 14 & $33 \%$ \\
\hline $\begin{array}{l}\text { Cuando se } \\
\text { obtiene de } \\
\text { revistas } \\
\text { científicas }\end{array}$ & $\begin{array}{l}\text { "Cuando se encuentra en páginas oficiales de } \\
\text { informes científicos publicados en revistas de } \\
\text { investigación y cuando se lo encuentra en } \\
\text { internet" }\end{array}$ & 6 & $5 \%$ & 1 & $2 \%$ \\
\hline $\begin{array}{l}\text { Cuando puede } \\
\text { ser verificado o } \\
\text { comprobado }\end{array}$ & $\begin{array}{l}\text { "De acuerdo a la cantidad de evidencia, } \\
\text { investigación y hechos acerca del conocimiento. } \\
\text { Mientras más se investigue y recolecte } \\
\text { información sobre un tema, más confiable será el } \\
\text { conocimiento científico" }\end{array}$ & 43 & $39 \%$ & 13 & $30 \%$ \\
\hline $\begin{array}{l}\text { Argumento de } \\
\text { autoridad }\end{array}$ & $\begin{array}{l}\text { "Cuando proviene de fuentes seguras, cuando los } \\
\text { científicos la aceptan y avalan. }\end{array}$ & 13 & $12 \%$ & 1 & $2 \%$ \\
\hline $\begin{array}{l}\text { Dedicación } \\
\text { personal }\end{array}$ & $\begin{array}{l}\text { "Cuando se pone mucho énfasis para realizar un } \\
\text { trabajo científico y es llevado a cabo por personas } \\
\text { que estudian y dedican mucho tiempo a esa } \\
\text { investigación" }\end{array}$ & 2 & $2 \%$ & 0 & 0 \\
\hline $\begin{array}{l}\text { Recorte de su } \\
\text { realidad }\end{array}$ & $\begin{array}{l}\text { "Cuando cada autor hace un recorte de su realidad. } \\
\text { Sólo podemos confiar en el conocimiento luego de } \\
\text { que nosotros leemos sobre él y sacamos nuestra } \\
\text { propia conclusión" }\end{array}$ & 0 & 0 & 2 & $5 \%$ \\
\hline $\begin{array}{l}\text { Imprecisa/incom } \\
\text { pleta }\end{array}$ & $\begin{array}{l}\text { "Se asume que el conocimiento científico es de } \\
\text { alguna forma confiable, pero no se presentan } \\
\text { justificaciones" }\end{array}$ & 23 & $21 \%$ & 9 & $21 \%$ \\
\hline $\begin{array}{l}\text { No es tan } \\
\text { confiable pues se } \\
\text { puede cambiar }\end{array}$ & $\begin{array}{l}\text { "Un conocimiento científico no es tan confiable ya } \\
\text { que pueden aparecer nuevas evidencias que } \\
\text { cambien el conocimiento anterior" }\end{array}$ & 2 & $2 \%$ & 1 & $2 \%$ \\
\hline \multirow[b]{2}{*}{ Otros } & $\begin{array}{l}\text { "Cuando es verificado por trabajos científicos } \\
\text { anteriores y se pueda establecer una comparación" }\end{array}$ & 2 & $2 \%$ & 2 & $5 \%$ \\
\hline & $\begin{array}{l}\text { "En un } 90 \% \text { me parece confiable cuando proviene } \\
\text { de universidades" } \\
\text { "Desconfío un poco cuando lo generan } \\
\text { laboratorios o institutos, ya que pienso que sus } \\
\text { intereses pueden estar involucrados" }\end{array}$ & 1 & $1 \%$ & 0 & 0 \\
\hline
\end{tabular}


Cuadro 3. ¿En qué medida es cambiante un conocimiento científico?

\begin{tabular}{|c|c|c|c|c|c|}
\hline \multirow[t]{2}{*}{ Categoría } & \multirow[t]{2}{*}{ Explicación } & \multicolumn{2}{|c|}{$\begin{array}{c}\text { UNC } \\
\text { (Argentina) }\end{array}$} & \multicolumn{2}{|c|}{$\begin{array}{c}\text { UFPR } \\
\text { (Brasil) }\end{array}$} \\
\hline & & $\mathbf{f}$ & $\%$ & $\mathbf{f}$ & $\%$ \\
\hline $\begin{array}{l}\text { Nunca está } \\
\text { acabado }\end{array}$ & $\begin{array}{l}\text { "El conocimiento científico no es definitivo pues } \\
\text { nunca está acabado. Nuevos conocimientos llevan } \\
\text { a la evolución de la ciencia" }\end{array}$ & 37 & $33 \%$ & 8 & $\begin{array}{l}21 \\
\%\end{array}$ \\
\hline $\begin{array}{l}\text { Puede ser } \\
\text { refutado }\end{array}$ & $\begin{array}{l}\text { "El conocimiento científico no está exento de } \\
\text { error y puede ser refutado por nuevas } \\
\text { investigaciones" } \\
\text { "Cuando no se encuentra elaborado } \\
\text { correctamente" } \\
\text { "Dependiendo del diseño metodológico que se } \\
\text { aplique durante la investigación" }\end{array}$ & 29 & $26 \%$ & 19 & $\begin{array}{l}50 \\
\%\end{array}$ \\
\hline $\begin{array}{l}\text { Porque está } \\
\text { vinculado a la } \\
\text { utilidad del } \\
\text { conocimiento }\end{array}$ & $\begin{array}{l}\text { "Varía según los fines para los que se lleva a } \\
\text { cabo" } \\
\text { "Es cambiante cuando la sociedad comienza a } \\
\text { hacer algo diferente, ya sea por tiempo, dinero o } \\
\text { interés" }\end{array}$ & 6 & $5 \%$ & 0 & 0 \\
\hline $\begin{array}{l}\text { Cambia según } \\
\text { perspectivas }\end{array}$ & $\begin{array}{l}\text { "Puede ser muy cambiante de acuerdo a la } \\
\text { sociedad en la que se produce ese conocimiento" } \\
\text { "Varía todo el tiempo, dependiendo del ambiente } \\
\text { donde se trabaje" }\end{array}$ & 5 & $4 \%$ & 0 & 0 \\
\hline $\begin{array}{l}\text { Surgimiento } \\
\text { de nuevas } \\
\text { tecnologías }\end{array}$ & $\begin{array}{l}\text { "Cambia a medida que surgen nuevas técnicas de } \\
\text { estudio y tecnologías" }\end{array}$ & 16 & $14 \%$ & 2 & $5 \%$ \\
\hline $\begin{array}{l}\text { Tiempo como } \\
\text { variable de } \\
\text { cambio }\end{array}$ & $\begin{array}{l}\text { "Los datos de la investigación pueden cambiar a } \\
\text { lo largo del tiempo" }\end{array}$ & 3 & $3 \%$ & 4 & $\begin{array}{l}11 \\
\%\end{array}$ \\
\hline $\begin{array}{l}\text { Cambia en } \\
\text { poca medida }\end{array}$ & $\begin{array}{l}\text { "Generalmente un conocimiento científico está } \\
\text { validado como correcto, por lo que su cambio es } \\
\text { poco probable" }\end{array}$ & 2 & $2 \%$ & 0 & 0 \\
\hline Imprecisa & $\begin{array}{l}\text { "Algunos conocimientos son cambiantes y otros } \\
\text { son estáticos" } \\
\text { "Un conocimiento científico cambia muchas veces } \\
\text { cuando se modifica el entorno en el cual se } \\
\text { encuentra" } \\
\text { "Una vez que se comienza a aplicar y da buenos } \\
\text { resultados" }\end{array}$ & 13 & $12 \%$ & 3 & $8 \%$ \\
\hline No sabe & ---- & 1 & $1 \%$ & 2 & $5 \%$ \\
\hline Otros & $\begin{array}{l}\text { "Cuando se basa en hechos espirituales o } \\
\text { personales" }\end{array}$ & 1 & $1 \%$ & 0 & 0 \\
\hline
\end{tabular}

UNC: Universidad Nacional de Córdoba. UFPR: Universidad Federal de Paraná. 
Cuadro 4. ¿Considera que haber cursado esta asignatura en el segundo cuatrimestre de primer año es el momento adecuado de la carrera?

\begin{tabular}{|c|c|c|c|c|c|}
\hline \multirow[t]{2}{*}{ Categoría } & \multirow[t]{2}{*}{ Explicación } & \multicolumn{2}{|c|}{$\begin{array}{c}\text { UNC } \\
\text { (Argentina) }\end{array}$} & \multicolumn{2}{|c|}{$\begin{array}{c}\text { UFPR } \\
\text { (Brasil) }\end{array}$} \\
\hline & & f & $\%$ & $\mathbf{f}$ & $\%$ \\
\hline $\begin{array}{l}\text { Mejora las } \\
\text { estrategias de } \\
\text { aprendizaje }\end{array}$ & $\begin{array}{l}\text { "Sí, porque mejora la manera de estudiar y } \\
\text { adquirir nuevos conocimientos" }\end{array}$ & 41 & $39 \%$ & 7 & $16 \%$ \\
\hline $\begin{array}{l}\text { Prepara para } \\
\text { realizar } \\
\text { investigaciones }\end{array}$ & $\begin{array}{l}\text { "Es el momento para formarnos como futuros } \\
\text { investigadores, ya que en la práctica } \\
\text { odontológica debemos investigar diversos casos } \\
\text { clínicos" }\end{array}$ & 17 & $16 \%$ & 2 & $5 \%$ \\
\hline $\begin{array}{l}\text { Hay necesidad } \\
\text { de profundizar } \\
\text { en otros espacios } \\
\text { curriculares }\end{array}$ & $\begin{array}{l}\text { "Creo que sí, pero debería retomarse en los } \\
\text { últimos años con mayor profundidad" }\end{array}$ & 2 & $2 \%$ & 11 & $25 \%$ \\
\hline $\begin{array}{l}\text { Se aprende el } \\
\text { lenguaje } \\
\text { científico que } \\
\text { permitirá la } \\
\text { lectura de } \\
\text { artículos }\end{array}$ & $\begin{array}{l}\text { "Sí, ya que en los próximos años de la facultad } \\
\text { estaremos capacitados para entender trabajos } \\
\text { científicos si las asignaturas lo requieren." } \\
\text { "Traje un pensamiento más crítico" }\end{array}$ & 2 & $2 \%$ & 14 & $32 \%$ \\
\hline \multirow[b]{3}{*}{$\begin{array}{l}\text { No la consideran } \\
\text { importante }\end{array}$} & $\begin{array}{l}\text { "El contenido de esta asignatura y el tiempo } \\
\text { requerido para las otras no permiten dedicación a } \\
\text { ésta" }\end{array}$ & 3 & $3 \%$ & 0 & $0 \%$ \\
\hline & $\begin{array}{l}\text { "Si estuviera en el primer cuatrimestre no se le } \\
\text { daría gran importancia" }\end{array}$ & 1 & $1 \%$ & 0 & $0 \%$ \\
\hline & $\begin{array}{l}\text { "Está bien ubicada, ya que esta asignatura no es } \\
\text { importante y no dificulta el cursado de otras, por } \\
\text { lo que es adecuado que esté en primer año; si } \\
\text { estuviera más adelante, con otras asignaturas por } \\
\text { cursar, podría tornase un obstáculo; podría estar } \\
\text { en cualquier momento" } \\
\text { "Sí, porque son conocimientos básicos" }\end{array}$ & 4 & $4 \%$ & 0 & $0 \%$ \\
\hline $\begin{array}{l}\text { Introduce a la } \\
\text { carrera de } \\
\text { Odontología }\end{array}$ & $\begin{array}{l}\text { "Es una base fundamental para formarse como } \\
\text { profesional de la salud y tener mayor } \\
\text { conocimiento sobre los temas y problemas que } \\
\text { ocurren en nuestra sociedad" } \\
\text { "Sí, porque al comenzar esta carrera debemos } \\
\text { tener una mirada más general; es una de las } \\
\text { asignaturas que va forjando una visión general } \\
\text { del futuro odontólogo" } \\
\text { "Ayuda en el proceso de incorporación y } \\
\text { adaptación a la facultad y a la carrera" }\end{array}$ & 9 & $8 \%$ & 0 & $0 \%$ \\
\hline $\begin{array}{l}\text { Desarrolla el } \\
\text { razonamiento } \\
\text { clínico }\end{array}$ & $\begin{array}{l}\text { "Sí, ya que brinda un análisis del conocimiento de } \\
\text { manera metódica y ordenada que será útil en la } \\
\text { evaluación de los aspectos clínicos del paciente" }\end{array}$ & 4 & $4 \%$ & 0 & $0 \%$ \\
\hline
\end{tabular}




\begin{tabular}{|c|c|c|c|c|c|}
\hline \multirow[t]{2}{*}{ Categoría } & \multirow[t]{2}{*}{ Explicación } & \multicolumn{2}{|c|}{$\begin{array}{c}\text { Cuadro } 4 . \\
\text { UNC } \\
\text { (Argentina) }\end{array}$} & \multicolumn{2}{|c|}{$\begin{array}{l}\text { ontinuación } \\
\text { UFPR } \\
\text { (Brasil) }\end{array}$} \\
\hline & & $\mathbf{f}$ & $\%$ & $\mathbf{f}$ & $\%$ \\
\hline $\begin{array}{l}\text { Debería iniciar } \\
\text { en el primer } \\
\text { cuatrimestre }\end{array}$ & $\begin{array}{l}\text { "Esta asignatura nos serviría, en cierto modo, para } \\
\text { proceder ante un problema y podría iniciar antes en } \\
\text { la carrera" } \\
\text { "Se debería cursar en el primer cuatrimestre de la } \\
\text { carrera y, quizás, aplicarla luego en las demás } \\
\text { asignaturas a través de un trabajo final" } \\
\text { "...podría aprender más sobre investigación, ya } \\
\text { que tal como se dicta la asignatura en la actualidad, } \\
\text { no me siento segura para hacer un trabajo de } \\
\text { investigación" }\end{array}$ & 7 & $7 \%$ & 0 & $0 \%$ \\
\hline \multirow{5}{*}{$\begin{array}{l}\text { Debería iniciar a } \\
\text { partir del tercer } \\
\text { cuatrimestre }\end{array}$} & $\begin{array}{l}\text { "No, debería estar más adelante en la carrera, pues } \\
\text { su contenido es muy odontológico y estaría mejor } \\
\text { ubicada cerca de la actividad clínica a fin de } \\
\text { recordarlos" }\end{array}$ & 6 & $6 \%$ & 3 & $7 \%$ \\
\hline & $\begin{array}{l}\text { "No vemos la importancia de la asignatura, ya que } \\
\text { estamos comenzando a formar hábitos de estudio y } \\
\text { a aprender a estudiar" } \\
\text { "No tenemos conocimientos científicos como para } \\
\text { entender la importancia de una investigación, y por } \\
\text { ello no nos atrae" }\end{array}$ & 2 & $2 \%$ & 0 & $0 \%$ \\
\hline & $\begin{array}{l}\text { "No me parece adecuado en este momento ya que } \\
\text { no estoy preparado a nivel universitario como para } \\
\text { cursar tres asignaturas difíciles (Anatomía, } \\
\text { Histología y Química Biológica); además me } \\
\text { parece una pérdida de tiempo" }\end{array}$ & 2 & $2 \%$ & 0 & $0 \%$ \\
\hline & $\begin{array}{l}\text { "No, a esta edad no le damos mucha importancia } \\
\text { ya que no sabemos a qué nos vamos a dedicar una } \\
\text { vez egresados" } \\
\text { "No, debería estar en los últimos años porque no } \\
\text { todos van dedicarse a la investigación" }\end{array}$ & 1 & $1 \%$ & 0 & $0 \%$ \\
\hline & $\begin{array}{l}\text { "Debería estar más próxima a la preparación del } \\
\text { trabajo final de la carrera" }\end{array}$ & --- & --- & 6 & $14 \%$ \\
\hline $\begin{array}{l}\text { Conocimiento } \\
\text { sobre } \\
\text { oportunidades } \\
\text { de investigación }\end{array}$ & $\begin{array}{l}\text { "Fui informada/o por la universidad acerca de } \\
\text { que existen varios proyectos de investigación" }\end{array}$ & 41 & $39 \%$ & 37 & $\begin{array}{l}100 \\
\%\end{array}$ \\
\hline $\begin{array}{l}\text { Interés en } \\
\text { realizar } \\
\text { actividades de } \\
\text { investigación }\end{array}$ & $\begin{array}{l}\text { "Me gustaría aprender a elaborar un informe de } \\
\text { investigación y experimentar la investigación en } \\
\text { la práctica" }\end{array}$ & 63 & $46 \%$ & 33 & $65 \%$ \\
\hline Otros & $\begin{array}{l}\text { "Lo mejor es comenzar cuanto antes con este } \\
\text { tipo de asignaturas" }\end{array}$ & 4 & $4 \%$ & 1 & $2 \%$ \\
\hline
\end{tabular}


al desarrollo de un país y a mejorar las condiciones de vida de las personas. Además, consideran que el conocimiento científico es dinámico, refutable, nunca acabado, siendo más alta la identificación de estos rasgos en los alumnos de la UFPR. El reconocimiento de dichos rasgos es satisfactorio para el nivel de formación de los alumnos.

Por otra parte, los estudiantes no consideran de fundamental importancia a estos conocimientos y al desarrollo del razonamiento clínico para el futuro ejercicio profesional, aunque en los contenidos de las asignaturas se explicita la importancia del conocimiento científico para la odontología, así como también la odontología basada en la evidencia. Esta última debería ser el norte de la actuación clínica y la investigación no debería quedar solamente a nivel teórico ${ }^{11}$. Es importante tener en cuenta que estos alumnos de primer año de la carrera no han realizado aún actividades académicas relacionadas con la práctica clínica. Quizás esta sea la razón por la que los estudiantes expresan que la formación científica sólo es necesaria para quienes se dediquen a la investigación. Según Depaola $(2002)^{14}$, las ciencias básicas y sociales pueden ser percibidas como obstáculos que los alumnos necesitan superar para alcanzar la etapa de la enseñanza clínica, en la que habría escasas oportunidades para la aplicación de estos conocimientos, cuando los currículos no están adecuadamente integrados.

Algunos autores sostienen que el propósito de la educación odontológica es formar a un profesional que no sólo posea los conocimientos técnicos necesarios para la atención del paciente, sino también, e igualmente importante, que pueda pensar críticamente, comprender el método científico y aplicarlo en la práctica profesional ${ }^{2,5,14}$. Sin embargo, no se exige a los alumnos de grado el uso suficiente de literatura científica, por lo que es necesario concientizarlos acerca de la variedad y el dinamismo del conocimiento actual, lo que implica poner al alumno en situaciones de aprendizaje en las que pueda realizar lectura crítica y aplicación del pensamiento científico ${ }^{11}$ para que, de esa manera, se puedan lograr las líneas directrices de los planes de estudio de las carreras de Odontología de UFPR y UNC.

Casi la mitad de los alumnos en este estudio no demostraron interés por realizar tareas de investigación en la facultad debido a que prefieren dedicarse a otras actividades. Según Depaola (2002), esa falta de interés puede deberse a no comprender el valor de la investigación en cualquier área de la salud ${ }^{14}$.

Un alto porcentaje de alumnos de las dos universidades acuerdan con la ubicación actual de las asignaturas, mientras que algunos de ellos particularmente de la UFPR- la ubicarían en etapa más avanzada de la carrera, sobre todo atendiendo a la necesidad de llevar a cabo el trabajo final. Sin embargo, la inclusión de metodología científica significó un aporte a su proceso de estudio en cuanto a trabajo metódico y rigor intelectual. Es importante tener en cuenta que, en ambas universidades, este es el único espacio curricular cuyo objeto de estudio es el conocimiento científico.

En este aspecto, Amâncio (2004) ${ }^{1}$ sostiene que las asignaturas sobre metodología científica deberían plantearse en el primer año de la carrera, en tanto que otros autores sugieren que sean incluidas más tempranamente, es decir en la enseñanza media. Además, otros investigadores consideran que es insuficiente una única asignatura que brinde el conocimiento científico y que la universidad debería ofrecer una secuencia que sustente la formación en investigación y oportunidades de experiencia práctica $^{5,15,16}$. Por lo tanto, al ser incluida en el inicio de la carrera, la metodología científica debería ir profundizándose de manera gradual a través de los años de formación, aplicando los principios de continuidad y complejidad creciente y con congruencia vertical y horizontal en el currículo ${ }^{15,16}$.

Se han observado marcadas diferencias entre las dos universidades en cuanto al conocimiento 
acerca de las oportunidades para desarrollar actividades de investigación, seguramente debido a la futura realización del trabajo final de la carrera en la UFPR y la posibilidad de obtener becas para llevarlo a cabo.

Estudios realizados en países desarrollados describen el impacto a largo plazo en los graduados de Facultades de Odontología que han tenido experiencias de investigación en el currículo ${ }^{17}$. La presencia constante de investigación durante la carrera puede contribuir a aumentar la productividad e inducir cambios en la actitud de alumnos y docentes ${ }^{18}$. Un estudio llevado a cabo en seis Escuelas Médicas de Brasil, con estudiantes de sexto año, mostró que ellos sólo pudieron comprender las ventajas de la investigación una vez que se involucraron en una actividad de ese tipo y afirmaron que, a pesar de las dificultades con los aspectos científicos, estarían interesados en integrar equipos de investigación aunque no tengan como objetivo dedicarse a esta tarea ${ }^{19}$.

Entre las limitaciones de este trabajo, se debe tener en cuenta que, por tratarse de un estudio transversal, no se pudieron reconocer sus conocimientos $\mathrm{o}$ experiencias previas en investigación ni los posibles cambios en sus opiniones con posterioridad al cursado de metodología científica. Además, se debe considerar el desigual tamaño de las muestras debido a las diferencias en las condiciones de ingreso (irrestricto en UNC y restringido en UFPR), lo que determina un número disímil de estudiantes que cursan la carrera.

\section{CONCLUSIÓN}

Si bien se ha detectado el inicio de la formación del pensamiento científico en alumnos de primer año de la carrera de Odontología de las dos universidades analizadas, se requiere un desarrollo más completo y complejo de esta competencia para el logro de las líneas directrices establecidas por las dos universidades para un profesional odontólogo. Esto implicaría una formación gradual durante toda la carrera a través de otros espacios curriculares, así como de metodologías de aplicación de la evidencia científica en las otras asignaturas.

\section{RESUMO \\ Desenvolvimento do pensamento científico em estudantes de Odontologia do primeiro ano de duas universidades latinoamericanas}

O objetivo deste estudo foi analisar o desenvolvimento do pensamento científico em estudantes do primeiro ano da graduação em Odontologia em duas universidades latinoamericanas (Brasil e Argentina), a partir da inclusão da disciplina de metodologia científica no currículo. A amostra foi composta por 144 estudantes. Embora o início da formação do pensamento científico tenha sido identificado nos alunos que compuseram a amostra, é necessário um desenvolvimento mais completo, gradual e complexo dessa competência para o alcance das diretrizes estabelecidas pelas duas universidades para um profissional cirurgião dentista.

Descritores: Metodologia Científica. Educação. Associação de Universidades do Grupo Montevidéu.

\section{ABSTRACT \\ Development of scientific thinking in first-year Dentistry students at two Latin-American universities}

The aim of this study was to analyze the development of scientific thought in undergraduate first year students, who participated of a scientific methodology classroom at the beginning of the dental career in two Latin American universities (Brazil y Argentina). The sample consisted of 144 students. Although the inclusion of the training process for scientific thought in first year students of the dental careers of the two universities analyzed was detected, a more complete, gradual and complex competence development is required for the achievement of established guidelines by the two universities for a professional dentist.

Descriptors: Scientific Methodology. Education. Asociación de Universidades del Grupo 
Montevideo.

\section{REFERENCIAS}

1. Amâncio AM. Inserção e atuação de jovens estudantes no ambiente científico: interação entre ensino e pesquisa. Escola Nacional de Saúde Pública Sergio Arouca, 2004.

2. Merideidy PV, Suarez MG, Moreno CAC. Actitud en estudiantes de Ciencias de la Salud hacia el conocimiento científico. Rev Cienc Salud. 2013;11(1):83-91.

3. UNESCO. Declaração de Santo Domingo: a ciência para o século XXI; uma visão nova e uma base de ação. Conferência Mundial sobre Ciências. Santo Domingo,1999.

4. Pineda EB, Canales FH, Alvarado EL. La enseñanza del metodo cientifico en la formación basica del profesional de salud. Educ Med Salud. 1987;21(3):243-55.

5. Pérez EGB. La investigación educacional: una necesidad para el desarrollo. Rev Medica Electron. 2011;33(2):252-3.

6. Cervo AL, Bervian PA. Metodologia científica. 3. ed. Braga JM, editor. São Paulo: McGraw-Hill; 1983. 3-20 p.

7. Lakatos EM, Marconi M de A. Metodologia Científica. 1 ed. São Paulo: Atlas, 1986.

8. Yuni JA, Urbano CA. Técnicas para Investigar: recursos metodológicos para la preparación de proyectos de investigación. 1 ed. Córdoba: Brujas, 2014.

9. Moreno X. Research training in dental undergraduate curriculum in Chile. J Oral Res.
2014;3(2):95-9.

10. Asociación de Universidades Grupo Montevideo. Programa ESCALA de Estudiantes de Posgrado de AUGM. 2016 Available from: http://grupomontevideo.org

11. Shirahatti R V, Sura S, Sumanthprasad GR, Khurana L. Dental students research inventory: a questionnaire to assess research challenges and opportunities. J Dent Educ. 2010;74(12):1308-18.

12. Brasil. Ministério da Educação. Diretrizes Curriculares Nacionais do curso de graduação em Odontologia. Brasília: Diário Oficial da União, 2002.

13. Brasil. Ministério da Educação. Resolução CNE/CES 3: Diretrizes Curriculares Nacionais do Curso de Graduação em Odontologia. Diário Oficial da União. Brasília, 2002.

14. Depaola D, Howell H, Baker CG, Boy-lefevre ML, Hull P, Holmstrup P, et al. Research and the Dental student. Eur J Dent Educ. 2002;6(3):45-51.

15. Pineda EB, Canales FH, Alvarado ELS. La enseñanza del método científico en la formación básica del profesional de salud. Educ Med Salud. 1987;21(3):243-55.

16. Cassiani SH de B, Rodrigues LP. O ensino da metodologia científica em oito escolas de Enfermagem da região Sudeste. Rev Latinoam Enfermagem. 1998;6(2):73-81.

17. Scott JE, Vries J, Iacopino AM. 25-year 
Analysis of a Dental undergraduate research training program (BSc Dent) at the University of Manitoba Faculty of Dentistry. J Dent Res. 2008;87(12):1085-8.

18. Iacopino AM, Pryor ME. The Effect of NIDCR R25 Grant Support on the curriculum and culture of a research non-intensive. J Dent Res. 2007;86(7):581-5.
19. Oliveira NA, Alves LA, Luz MR. Iniciação Científica na graduação: o que diz o estudante de Medicina?. Rev Bras Educ Med. 2008;32(3):309-14.

\section{Correspondencia para:}

Allana Pivovar

e-mail: lana.pivovar@gmail.com

Av. Pref. Lothário Meissner, 632 - Jardim Botânico 80210-170 Curitiba/PR Brasil 